\title{
Risk-stratification in febrile infants 29 to 60 days old: a cost-effectiveness analysis
}

\author{
Kathleen A. Noorbakhsh ${ }^{1 *}$, Sriram Ramgopal ${ }^{2}$, Nancy S. Rixe ${ }^{1}$, Jennifer Dunnick ${ }^{1}$ and Kenneth J. Smith ${ }^{3}$
}

\begin{abstract}
Background: Multiple clinical prediction rules have been published to risk-stratify febrile infants $\leq 60$ days of age for serious bacterial infections (SBI), which is present in $8-13 \%$ of infants. We evaluate the cost-effectiveness of strategies to identify infants with SBI in the emergency department.

Methods: We developed a Markov decision model to estimate outcomes in well-appearing, febrile term infants, using the following strategies: Boston, Rochester, Philadelphia, Modified Philadelphia, Pediatric Emergency Care Applied Research Network (PECARN), Step-by-Step, Aronson, and clinical suspicion. Infants were categorized as low risk or not low risk using each strategy. Simulated cohorts were followed for 1 year from a healthcare perspective. Our primary model focused on bacteremia, with secondary models for urinary tract infection and bacterial meningitis. One-way, structural, and probabilistic sensitivity analyses were performed. The main outcomes were SBI correctly diagnosed and incremental cost per quality-adjusted life-year (QALY) gained.

Results: In the bacteremia model, the PECARN strategy was the least expensive strategy (\$3671, 0.779 QALYs). The Boston strategy was the most cost-effective strategy and cost $\$ 9799 /$ QALY gained. All other strategies were less effective and more costly. Despite low initial costs, clinical suspicion was among the most expensive and least effective strategies. Results were sensitive to the specificity of selected strategies. In probabilistic sensitivity analyses, the Boston strategy was most likely to be favored at a willingness-to-pay threshold of $\$ 100,000 / Q A L Y$. In the urinary tract infection model, PECARN was preferred compared to other strategies and the Boston strategy was preferred in the bacterial meningitis model.
\end{abstract}

Conclusions: The Boston clinical prediction rule offers an economically reasonable strategy compared to alternatives for identification of SBI.

Keywords: Infant fever, Economic analysis, Clinical prediction rules, Serious bacterial infection, Neonatal sepsis

\section{Background}

Fever is one of the most common chief complaints in the emergency department (ED), comprising $10-20 \%$ of all pediatric ED visits [1-3]. Among infants $\leq 60$ days old, the prevalence of serious bacterial infections (SBI), including bacterial meningitis, bacteremia and urinary

\footnotetext{
*Correspondence: Katie.Noorbakhsh@chp.edu

${ }^{1}$ Department of Pediatrics, University of Pittsburgh Medical Center,

Children's Hospital of Pittsburgh, 4401 Penn Ave, Pittsburgh, PA 15224

60611, USA

Full list of author information is available at the end of the article
}

tract infection (UTI), in the setting of fever ranges from 8 to $13 \%$ [4-9]. Such infections pose potential risk of morbidity and mortality if not diagnosed [4-9]. The evaluation and management of young febrile infants involves extensive diagnostic testing, frequently followed by hospitalization and antibiotic therapy [10].

.Balancing the costs of medical evaluation and treatment, particularly unnecessary hospitalization, with the risks of misdiagnosis poses a clinical challenge. Over the last 40 years, multiple clinical prediction rules have been published to identify a cohort of infants at low risk of SBI [11-17]. Older prediction rules require routine lumbar 
puncture and cerebrospinal fluid (CSF) testing [15-17]. More recent prediction rules do not require CSF testing for risk stratification and offer improved diagnostic accuracy [15-18]. These rules carry the potential to improve clinical outcomes, decrease variation in care, and reduce high costs associated with the evaluation and management of febrile infants [4, 7]. The cost-effectiveness of published clinical prediction rules in this population is unknown. The decision to adopt a clinical prediction rule to evaluate febrile infants must be weighed against the cost and effectiveness of established risk-stratification strategies.

In this investigation, we use decision modeling techniques to evaluate the cost-effectiveness of different strategies to identify infants with SBI in the ED.

\section{Methods}

\section{Study design}

We created a decision-analytic Markov model to simulate a hypothetical cohort of infants 29-60 days old presenting to an ED using the following eight published risk-stratification strategies: Boston, Rochester, Philadelphia, Modified Philadelphia, Pediatric Emergency Care Applied Research Network (PECARN), Step-by-Step, Aronson, and clinical suspicion (Table 1) [11-18]. Selection of strategies was limited to those developed to identify infants at low risk of SBI and with published sensitivity and specificity data. All analyses used secondary data from the medical literature or from online US databases without identifiable patient information. The decision model was programmed in TreeAge Pro 2016 (TreeAge Software, Inc., Williamstown, MA).

Our base case was a 40-day old term infant presenting to the ED with fever. A "base case" serves as the most likely scenario and lays the groundwork for model assumptions. All infants were assumed to be "wellappearing" and without localizing signs of infection. By selecting these characteristics, our base case infant met evaluation criteria and low-risk history criteria for each risk-stratification strategy. Infants < 29 days of age were excluded as most clinical prediction rules consider this age group to be inherently not low risk.

A Markov model consists of mutually exclusive "health states." Simulated individuals reside in one health state at a time and can transition between health states as designated in the model. For this study, we considered five health states: 1) well, 2) SBI with medical treatment, 3) misdiagnosed SBI with no medical treatment, 4) misdiagnosed well infant with medical treatment, and 5) death. Infants in the simulated population were initially categorized as low risk or not low risk using each strategy. Infants with SBI who were misidentified as low risk had an increased risk of death above baseline [29-32].
Those remaining alive underwent reevaluation and medical treatment. Infants who underwent medical treatment were assumed to make a full recovery. Infants without SBI who were miscategorized as not low risk were assumed to undergo hospitalization without complication. Low-risk criteria were defined according to each clinical prediction rule (Table 1). For clinical suspicion, low-risk was defined as a risk of SBI $<1 \%$ as determined by the treating physician and was assumed to be assigned after history and physical exam but before obtaining diagnostic testing [18]. The threshold of $1 \%$ was selected based on a previous study [18]. For the Aronson strategy, we used a score $<2$ to define low risk [14]. For the Boston strategy, empiric ceftriaxone administration for all lowrisk infants was assumed to be protective against worsening infection and death [16].

Due to significant variations in the reported prevalence [4-9], complications [8, 9, 30-36], and costs of treatment [37] for UTI, bacteremia, and bacterial meningitis, our primary model focused on bacteremia, with secondary models for UTI and bacterial meningitis. We chose bacteremia for our primary model, as it is more prevalent than bacterial meningitis and carries higher risks of morbidity and mortality than UTI [4-9, 30-36]. In the bacterial meningitis model, misdiagnosed infants with bacterial meningitis had an increased risk of death compared to the bacteremia model $[9,30-32]$. In the UTI model, misdiagnosed infants with UTI returned to the ED and were treated, with a small proportion developing bacteremia [33-35]. There was no increased risk of death in the UTI model [33]. The Aronson and Modified Philadelphia prediction rules do not report sensitivity or specificity for UTI and were not included in the UTI model. We constructed a decision tree for each model of interest. A simplified version of the bacteremia decision tree is presented in Fig. 1.

\section{Model input variables}

Input parameters for probabilities, costs, and outcomes are presented in Tables 1 and 2. For each variable, we included an estimated 95\% probability range. We conducted a review of published literature to identify rates of outcomes for febrile infants and to identify measures of diagnostic accuracy for prediction rules (Table 1). Probabilities of outcomes from misdiagnosed bacteremia were derived from previously published literature, with ranges that accounted for variation among sources and uncertainty given paucity of data in the post antibiotic and vaccine eras [31]. All-cause mortality was estimated using U.S. National Center for Health Statistics life tables [38].

Sensitivity and specificity of each clinical prediction rule for bacteremia, UTI, and bacterial meningitis, were hand calculated by two of the authors (KAN, SR) 


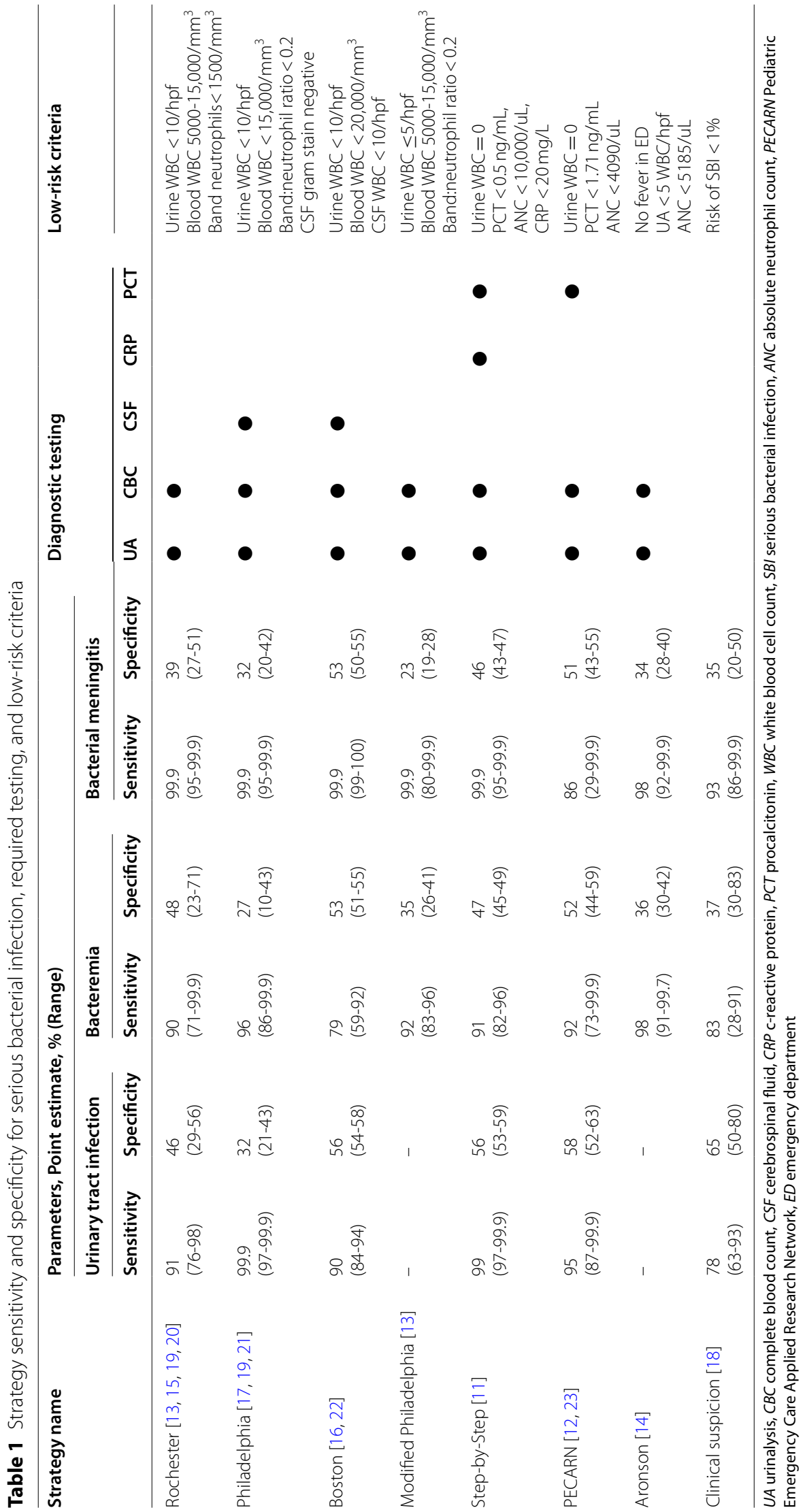




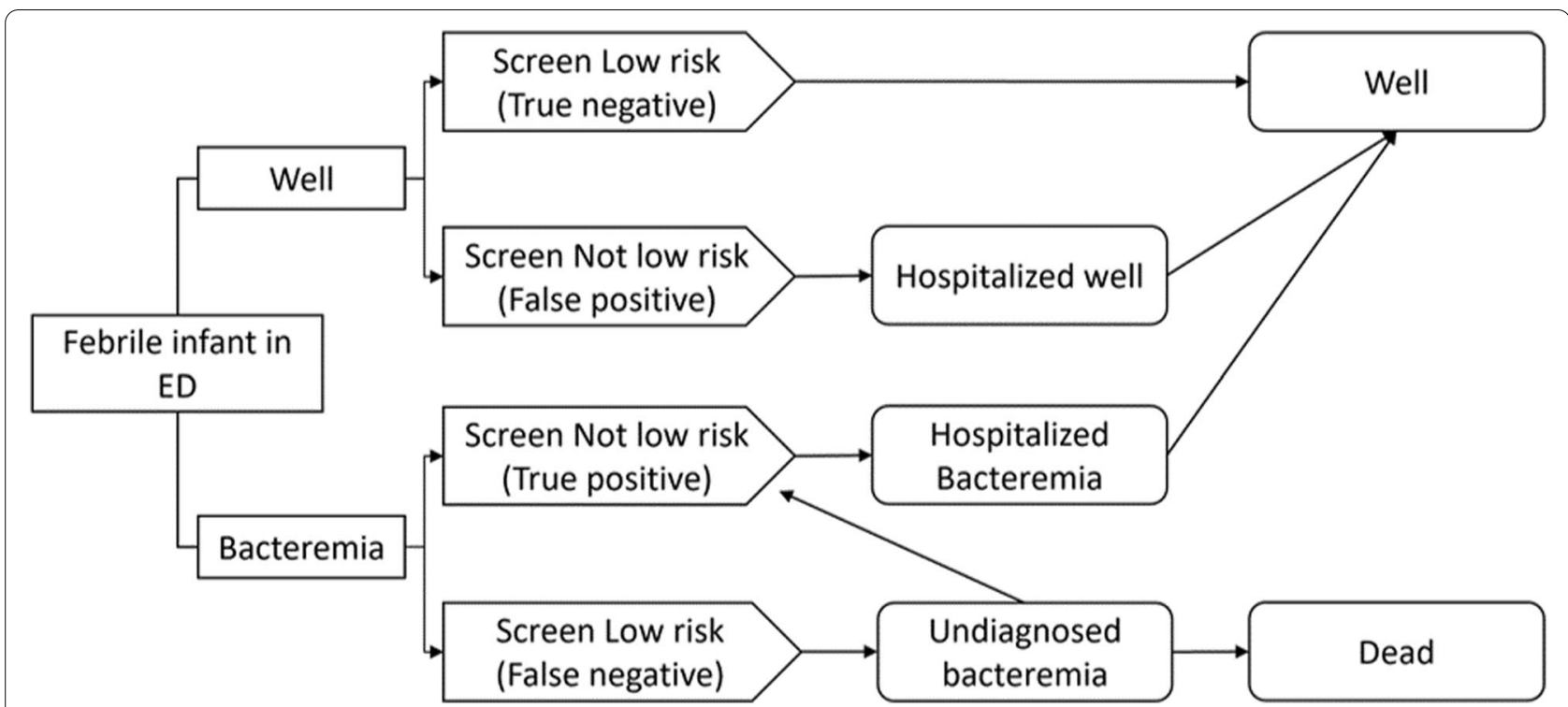

Fig. 1 Model schematic for bacteremia. Infants begin the model in the emergency department with a fever, either with or without bacteremia and undergo risk-stratification. Infants with bacteremia who are misidentified as low risk have an increased risk of death. ED, emergency department

based on published data [11-23]. When more than one study for a prediction rule was identified, the mean values for calculated sensitivity and specificity were used. Range was based on 95\% confidence interval for prediction rules with a single data source. For prediction rules with externally validated data, the range was broadened to include values from all calculated 95\% confidence intervals.

Costs included direct medical costs of ED visits, diagnostic testing, and medical treatment (Table 2). Costs of hospitalization were based on the mean value for each diagnosis for children less than a year of age, as reported by the Healthcare Cost and Utilization Project [37]. All costs were adjusted to 2016 US dollars based on the medical cost component of the Consumer Price Index [26]. We selected 2016 as this was the most recent year available for US inpatient healthcare data from the Healthcare Cost and Utilization Project [37].

Health state utilities were assigned a value of $0-1$, with 0 equivalent to death and 1 representing perfect health [28]. Utility values associated with various outcomes were drawn from the literature [24, 25, 39, 40]. When quality-of-life studies were not available for this age group, we used quality-of-life estimates from older populations. Infant mortality was factored as a lifetime disutility, meaning that the lifetime loss of qualityadjusted life-years (QALY) for each death was factored into the model. All costs and utilities were discounted at $3 \%$ per year, as recommended by the second Panel on Cost-Effectiveness in Health and Medicine [41].

\section{Cost-effectiveness analysis}

A cost-effectiveness analysis was conducted from a healthcare perspective, considering costs as they related directly to health expenditures, and run over a hypothetical one-year time horizon [41]. This differs from a societal perspective, which incorporates a comprehensive assessment of costs and benefits [41]. The primary outcomes evaluated in this study were cost, effectiveness (SBI accurately diagnosed and treated), and cost-effectiveness for each strategy. Strategies were ranked by cost then compared in terms of cost, effectiveness, and incremental cost-effectiveness ratio (ICER). The ICER measures added cost for additional benefit to a population, measured in QALYs, and reflects the value of an intervention. QALYs serve as a composite measure of morbidity and mortality. We assumed a willingness-to-pay of $\$ 100,000 /$ QALY gained, a commonly cited threshold for the US healthcare system [41]. A strategy was dominated by another strategy if it was both more costly and less effective. Preferred strategies were those with the highest ICER that did not exceed the willingness-to-pay threshold. Secondary outcomes included hospitalizations, lumbar punctures, and deaths. Findings are expressed as costs, QALYs gained, and cost per QALY gained.

\section{Sensitivity analyses}

We conducted one-way sensitivity analyses to determine if varying any individual parameter across its listed range substantially changed results. Threshold analyses determined the point at which changes to certain input 
Table 2 Bacteremia model inputs: Baseline parameter values and ranges

\begin{tabular}{|c|c|c|}
\hline Variable & Point estimate & (Range) \\
\hline \multicolumn{3}{|l|}{ Probabilities } \\
\hline Risk of bacteremia $[7,11-13,31]$ & $1.7 \%$ & $(0.01-3.7 \%)$ \\
\hline $\begin{array}{l}\text { Risk of death, delayed antibiotics } \\
\text { in bacteremia [29-31] }\end{array}$ & $10 \%$ & $(0-25 \%)$ \\
\hline \multicolumn{3}{|l|}{$\operatorname{Costs}^{a}$} \\
\hline ED visit [39] & $\$ 553$ & $(442-664)$ \\
\hline \multicolumn{3}{|l|}{ Hospitalization [37] } \\
\hline Infectious condition ruled out & $\$ 5550$ & $(4440-6660)$ \\
\hline Urinary tract infection & $\$ 5382$ & $(4305-6458)$ \\
\hline Bacteremia & $\$ 26,031$ & $(20,825-31,237)$ \\
\hline Meningitis & $\$ 29,464$ & $(23,571-35,357)$ \\
\hline Blood culture [27] & $\$ 12$ & $(10-14)$ \\
\hline CBC with differential [27] & $\$ 12$ & $(10-14)$ \\
\hline C-reactive protein [27] & $\$ 18$ & $(14-22)$ \\
\hline Procalcitonin [27] & $\$ 36$ & $(29-43)$ \\
\hline Urinalysis [27] & $\$ 4$ & $(3-5)$ \\
\hline Urine culture [27] & $\$ 11$ & $(9-13)$ \\
\hline Lumbar puncture [27] & $\$ 81$ & $(65-97)$ \\
\hline CSF culture [27] & $\$ 12$ & $(10-14)$ \\
\hline CSF gram stain [27] & $\$ 6$ & $(5-7)$ \\
\hline CSF testing, other [27] & $\$ 83$ & $(66-100)$ \\
\hline \multicolumn{3}{|l|}{ Utilities } \\
\hline Well newborn [39] & 0.95 & \\
\hline Hospitalization [39] & 0.88 & $(0.58-1.0)$ \\
\hline Bacteremia [24] & 0.71 & $(0.4-1.0)$ \\
\hline Lumbar puncture [25] & -0.1 & $\left(0.0-^{-} 0.5\right)$ \\
\hline Disutility of death [38] & 30.98 & \\
\hline
\end{tabular}

a Costs are in 2016 U.S. dollars

ED emergency department, CSF cerebrospinal fluid, $C B C$ complete blood count parameters (i.e. disease prevalence, sensitivity or specificity of each diagnostic strategy, or cost of medical management) resulted in a substantial change in the preferred strategy. Structural sensitivity analyses evaluated 1) the impact of empiric ceftriaxone administration in strategies that included testing of urine, blood, and CSF, and 2 ) the potential impact of contaminated cultures on the cost-effectiveness of each strategy. Probabilistic sensitivity analyses estimated the effect of uncertainties in each parameter. For the probabilistic sensitivity analyses, each variable was assigned a distribution of possible values. Distributions were chosen to reflect the level of certainty, the characteristics of the parameter range, and methodological standards. $\beta$ distributions were used for probabilities and quality adjustments; $\gamma$ distributions were used for costs. We then used the model to run 1000 simulations for each strategy. For each individual simulation, the model randomly selected a different value for each variable from its assigned distribution. Findings from the probabilistic sensitivity analysis are reported as costeffectiveness acceptability frontier curves [42]. These curves show the probability that the cost-effectiveness of optimal strategies will be less than or equal to a given $\$$ / QALY amount and reflect uncertainty in the model.

\section{Results}

\section{Bacteremia}

In the base-case analysis, the PECARN strategy was the least expensive (with a cost of $\$ 3671$, and a gain of 0.779 QALYs per individual). Compared to the PECARN strategy, the Boston strategy cost \$9799/QALY gained. All other strategies were dominated (Table 3). One-way sensitivity analyses demonstrated that the model was sensitive to mortality risk after misdiagnosis, bacteremia prevalence, and the sensitivity and specificity of PECARN, Modified Philadelphia, and Rochester strategies (Table 4, Fig. 2).

Table 3 Results of cost-effectiveness analyses, Bacteremia model

\begin{tabular}{llllll}
\hline & Cost $(\$)$ & Incremental Cost $\mathbf{\$}$ ) & Effectiveness (QALY) & $\begin{array}{l}\text { Incremental } \\
\text { Effectiveness(QALY) }\end{array}$ & $\begin{array}{l}\text { ICER (\$/QALY) } \\
\text { PECARN }\end{array}$ \\
Boston & $\$ 3671$ & - & 0.779 & - & - \\
Rochester & $\$ 3701$ & $\$ 30$ & $\mathbf{0 . 7 8 2}$ & $\mathbf{0 . 0 0 3}$ & $\mathbf{\$ 9 7 9 9}$ \\
Step-by-Step & $\$ 3846$ & $\$ 145$ & 0.778 & -0.004 & Dominated \\
Clinical suspicion & $\$ 3977$ & $\$ 276$ & 0.778 & -0.004 & Dominated \\
Aronson & $\$ 4430$ & $\$ 729$ & 0.764 & -0.019 & Dominated \\
Modified Philadelphia & $\$ 4527$ & $\$ 826$ & 0.782 & -0.001 & Dominated \\
Philadelphia & $\$ 4594$ & $\$ 892$ & 0.778 & -0.004 & Dominated \\
\hline
\end{tabular}

a Bold text: Favored strategy at a $\$ 100,000$ per quality-adjusted life-year threshold

${ }^{\mathrm{b}} \mathrm{A}$ dominated strategy is more costly and less effective than other strategies

QALY quality adjusted life years, ICER incremental cost-effectiveness ratio, PECARN Pediatric Emergency Care Applied Research Network 
Table 4 One-way sensitivity analysis results, Bacteremia model

\begin{tabular}{lllll}
\hline & & & \multicolumn{2}{l}{ Preferred Strategy } \\
\cline { 4 - 4 } Variable & Base-case & Threshold & Below threshold & Above threshold \\
\hline $\begin{array}{l}\text { Risk of bacteremia } \\
\text { Risk of death, delayed antibiotics }\end{array}$ & 0.017 & 0.005 & PECARN & Boston \\
$\begin{array}{l}\text { Strategy sensitivity } \\
\text { PECARN }\end{array}$ & 0.10 & 0.03 & PECARN & Boston \\
$\begin{array}{l}\text { Strategy specificity } \\
\text { Boston }\end{array}$ & $92 \%$ & $98 \%$ & Boston & PECARN \\
$\begin{array}{l}\text { PECARN } \\
\text { Rochester }\end{array}$ & $53 \%$ & $52 \%$ & PECARN & Boston \\
Clinical suspicion & $52 \%$ & $57 \%$ & Boston & PECARN \\
\hline
\end{tabular}

PECARN Pediatric Emergency Care Applied Research Network

\section{One-way sensitivity analysis: Bacteremia model PECARN vs Boston Strategies}

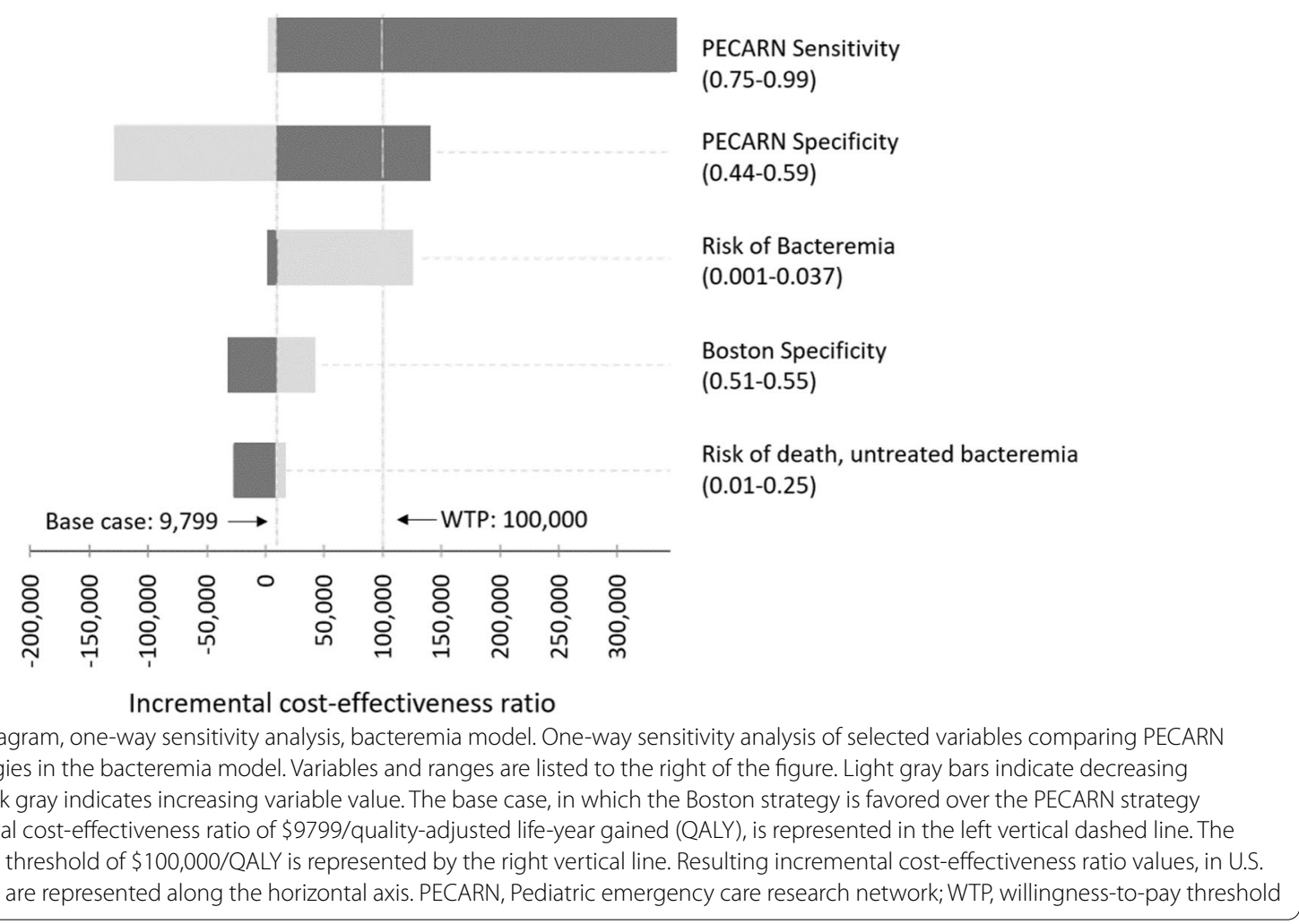

In a structural sensitivity analysis, when empiric ceftriaxone administration was removed from the Boston strategy, effectiveness of the Boston strategy decreased to 0.771 QALYs per individual. The PECARN strategy became the preferred strategy and Boston was dominated. Addition of ceftriaxone to the Philadelphia strategy did not change model outcomes. Addition of contaminated cultures with presumptive reevaluation and treatment of affected patients did not change model outcomes. Comparative clinical outcomes in a hypothetical population are shown in Table 5.

Probabilistic sensitivity analysis results are summarized as cost-effectiveness acceptability frontier curves, 
Table 5 Outcomes by strategy in a population of 1000 febrile infants, of whom 17 have bacteremia

\begin{tabular}{lllllll}
\hline Strategy & $\begin{array}{l}\text { Bacteremia } \\
\text { Cases, } \mathbf{n}\end{array}$ & $\begin{array}{l}\text { Not low risk, } \\
\text { Bacteremia, } \\
\%(\mathbf{n})\end{array}$ & $\begin{array}{l}\text { Low risk, } \\
\text { Bacteremia } \\
\%(\mathbf{n})\end{array}$ & $\begin{array}{l}\text { Not low risk, } \\
\text { No bacteremia, } \\
\text { \% (n) }\end{array}$ & $\begin{array}{l}\text { Lumbar Puncture, } \\
\%(\mathbf{n})\end{array}$ & $\begin{array}{l}\text { Death from } \\
\text { bacteremia, } \\
\%\end{array}$ \\
\hline Clinical suspicion & 17 & $66(11)$ & $31(6)$ & $62(620)$ & $63(633)$ & 0.05 \\
PECARN & 17 & $92(16)$ & $4(1)$ & $47(471)$ & $49(487)$ & 0.01 \\
Modified Philadelphia & 17 & $82(14)$ & $16(3)$ & $44(444)$ & $46(459)$ & 0.03 \\
Step-by-Step & 17 & $90(15)$ & $9(2)$ & $52(523)$ & $54(538)$ & 0.02 \\
Boston & 17 & $76(13)$ & $21(4)$ & $47(465)$ & $100(1000)$ & $<0.01$ \\
Rochester & 17 & $90(15)$ & $9(2)$ & $51(509)$ & $52(524)$ & 0.02 \\
Aronson & 17 & $97(17)$ & $3(0)$ & $63(631)$ & $65(648)$ & $<0.01$ \\
Philadelphia & 17 & $97(17)$ & $3(0)$ & $66(657)$ & $100(1000)$ & $<0.01$ \\
\hline
\end{tabular}

PECARN Pediatric Emergency Care Applied Research Network

showing the uncertainty associated with the optimal options, calculated using the net monetary benefit framework, over a range of willingness-to-pay (or acceptability) thresholds, as shown in Fig. 3. In this analysis, the Boston strategy was the preferred strategy when the willingnessto-pay was $>\$ 10,000 / \mathrm{QALY}$. At a willingness-to-pay of $\$ 100,000 / \mathrm{QALY}$, the Boston strategy was the more costeffective option in $20 \%$ of model iterations. A scatter-plot comparing the incremental cost and incremental effectiveness of the Boston strategy to the PECARN strategy demonstrated that the Boston strategy was below the $\$ 100,000 /$ QALY threshold in $67 \%$ of model iterations (Fig. 4).

PECARN, Pediatric Emergency Care Applied Research Network; WTP, willingness-to-pay threshold.
UTI

In the UTI model, the PECARN strategy was the least expensive strategy (\$3422, 0.842 QALYs). All other strategies were more expensive and less effective (Table 6).

One-way sensitivity analyses demonstrated that the Step-by-step strategy would be preferred if it had a specificity for UTI greater than $58 \%$ or if specificity of the PECARN strategy was less than $56 \%$. Clinical suspicion was preferred if it had a specificity greater than $67 \%$. Probabilistic sensitivity analyses indicated that at a threshold of $\$ 100,000 /$ QALY, the PECARN strategy was preferred in $48 \%$ of model iterations.

\section{Meningitis}

For bacterial meningitis, the PECARN strategy was least expensive, and the Boston strategy was preferred with an

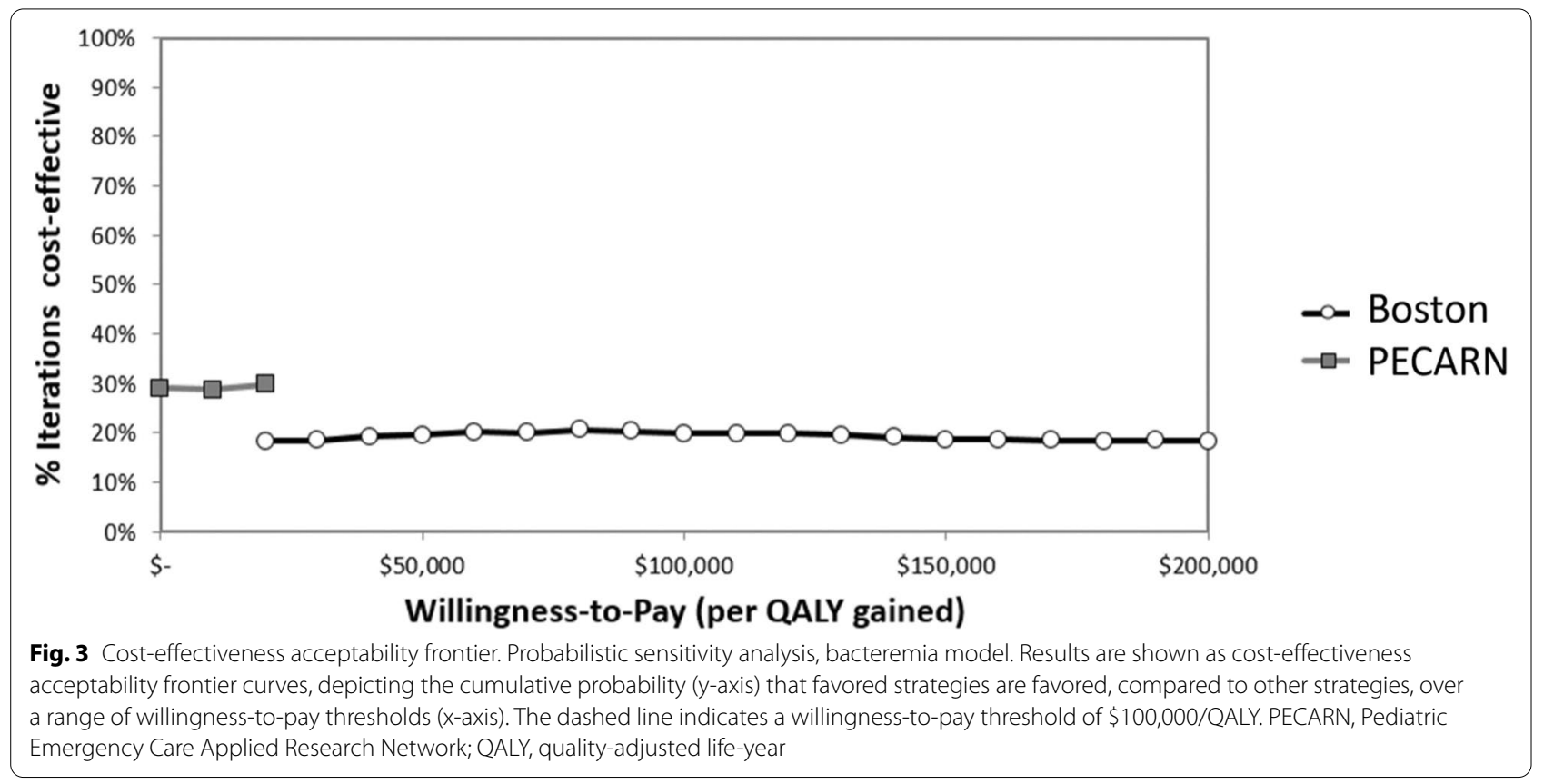


Probabilistic Sensitivity Analysis, PECARN vs. Boston

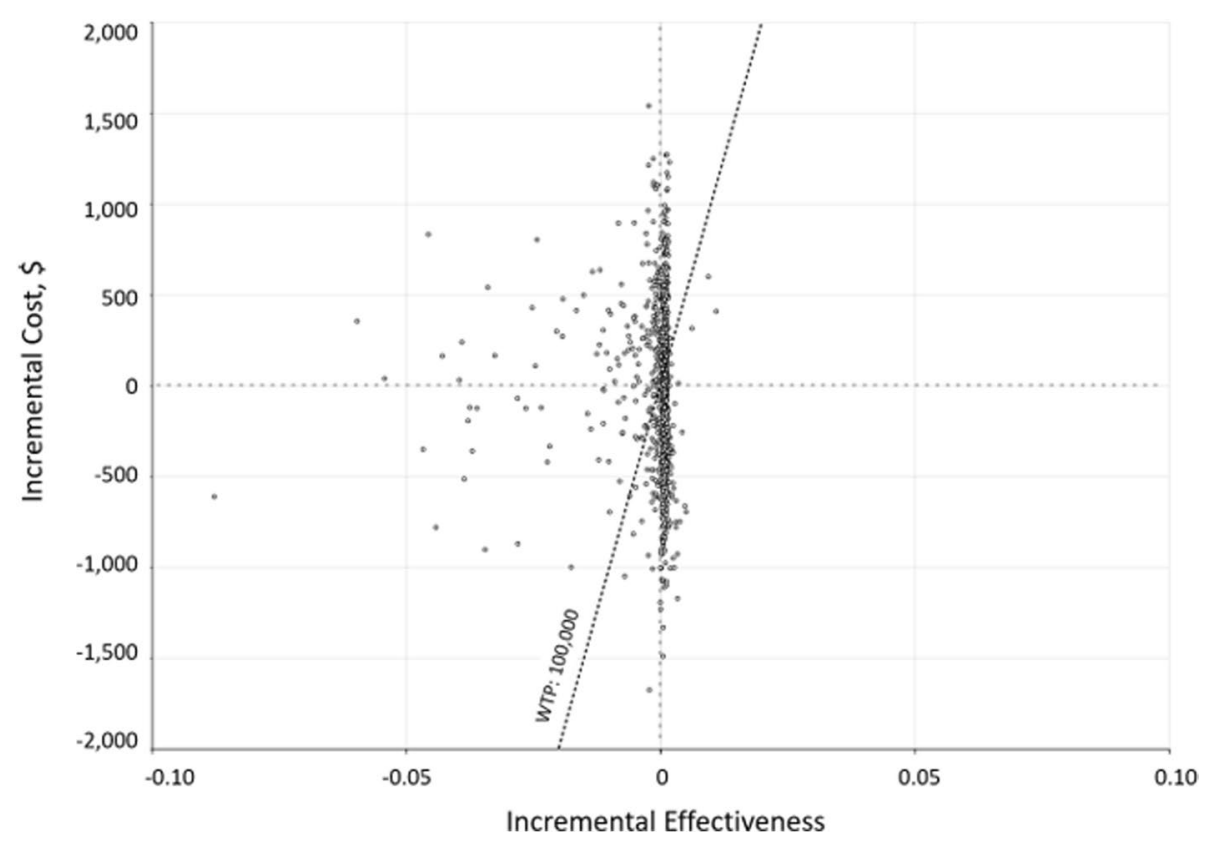

Fig. 4 Probabilistic sensitivity analysis, PECARN vs Boston, bacteremia model. Results of the probabilistic sensitivity analysis represented as a scatterplot of the incremental cost ( $x$-axis) and incremental effectiveness ( $y$-axis) of the Boston strategy as compared to the PECARN strategy. The willingness-to-pay threshold of $\$ 100,000$ /quality-adjusted life-year, is indicated by the diagonal dashed line

ICER of \$1198/QALY gained. All other strategies were dominated (Table 7). One-way sensitivity analyses demonstrated that this model was sensitive to the specificity of each clinical prediction rule. The PECARN strategy was favored if its sensitivity for identifying bacterial meningitis was greater than $99.8 \%$. Probabilistic sensitivity analysis indicated that at a threshold of $\$ 100,000 /$ QALY, the Boston strategy was preferred in $45 \%$ of model iterations.

\section{Discussion}

We performed a cost-effectiveness analysis to compare commonly cited strategies for risk-stratification in the evaluation of febrile infants, finding that Boston and PECARN strategies provided economically reasonable risk stratification strategies compared to other published clinical prediction rules. Models for each type of SBI varied with respect to treatment costs and health risks after misdiagnosis; the PECARN strategy was favored in the UTI model while the Boston strategy was increasingly cost-effective with higher risk infection types.

We found that the Boston strategy was the most costeffective strategy in both the bacteremia and bacterial meningitis models. Despite lower sensitivity compared to other strategies, the protective effect offered by empiric ceftriaxone and the cost-savings introduced by a higher specificity outweighed the disutility and costs associated with universal lumbar puncture and CSF testing.
Alternatively, the PECARN strategy, which does not require CSF testing or empiric antibiotic administration, was an economical option in both models and may provide benefits not measured in this study, depending on individual risk tolerance or preferences.

We found that in the UTI model, most strategies had a sensitivity $\geq 90 \%$ and the risks associated with delayed antibiotics were less substantial than in the other models. Because of this, the benefits of empiric ceftriaxone had a smaller impact, and the model became more sensitive to the costs and disutility associated with admitting patients who were ultimately not diagnosed with UTI.

We found that there were no scenarios in which application of clinical suspicion alone was economically reasonable. In this strategy, infants with fever were assumed to undergo no diagnostic testing if the treating physician assessed their risk to be $<1 \%$. Prior studies investigating variation in infant fever management have noted that a proportion of young febrile infants are discharged from pediatric EDs without additional testing [4]. Our model suggests that the benefits of decreased upfront resource utilization are outweighed by the increased risk and associated costs for the few missed infants with SBI.

To our knowledge, this is the first cost-effectiveness analysis of risk-stratification of febrile infants to evaluate the most recently reported clinical prediction rules for febrile infants. Lieu, et al., demonstrated the benefits of outpatient 
Table 6 Results of cost-effectiveness analyses, Urinary tract infection model

\begin{tabular}{llllll}
\hline & Cost $\mathbf{( \$ )}$ & Incremental Cost $\mathbf{( \$ )}$ & Effectiveness (QALY) & $\begin{array}{l}\text { Incremental Effectiveness } \\
\text { (QALY) }\end{array}$ & $\begin{array}{l}\text { ICER (\$/QALY) } \\
\text { PECARN }\end{array}$ \\
\hline Step-by-Step & $\$ 3422$ & - & $\mathbf{0 . 8 4 2}$ & - & - \\
Boston & $\$ 3505$ & $\$ 83$ & 0.842 & -0.0002 & Dominated $^{b}$ \\
Rochester & $\$ 3731$ & $\$ 310$ & 0.838 & -0.004 & Dominated \\
Clinical suspicion & $\$ 3995$ & $\$ 573$ & 0.841 & -0.001 & Dominated \\
Philadelphia & $\$ 4521$ & $\$ 1099$ & 0.837 & -0.005 & Dominated \\
\hline
\end{tabular}

a Bold text: Favored strategy at a $\$ 100,000$ per QALY

${ }^{\mathrm{b}} \mathrm{A}$ dominated strategy is more costly and less effective than other strategies

QALY quality adjusted life years, ICER incremental cost-effectiveness ratio, PECARN Pediatric Emergency Care Applied Research Network

Table 7 Results of cost-effectiveness analyses, Bacterial meningitis model

\begin{tabular}{|c|c|c|c|c|c|}
\hline & Cost (\$) & Incremental Cost (\$) & Effectiveness (QALY) & $\begin{array}{l}\text { Incremental Effectiveness } \\
\text { (QALY) }\end{array}$ & ICER (\$/QALY) \\
\hline PECARN & $\$ 4290$ & & 0.689 & & \\
\hline Boston & $\$ 4398$ & $\$ 108$ & 0.779 & 0.090 & $\$ 1198$ \\
\hline Step-by-Step & $\$ 4669$ & $\$ 272$ & 0.773 & -0.006 & Dominated \\
\hline Aronson & $\$ 5015$ & $\$ 617$ & 0.772 & -0.007 & Dominated \\
\hline Rochester & $\$ 5308$ & $\$ 911$ & 0.772 & -0.007 & Dominated \\
\hline Philadelphia & $\$ 5466$ & $\$ 1068$ & 0.771 & -0.008 & Dominated \\
\hline Clinical suspicion & $\$ 5518$ & $\$ 1120$ & 0.734 & -0.046 & Dominated \\
\hline Modified Philadelphia & $\$ 5824$ & $\$ 1426$ & 0.771 & -0.008 & Dominated \\
\hline
\end{tabular}

${ }^{a}$ Bold text: Favored strategy at a $\$ 100,000$ per quality adjusted life year threshold

${ }^{\mathrm{b}}$ A dominated strategy is more costly and less effective than other strategies

QALY quality adjusted life years, ICER incremental cost-effectiveness ratio, PECARN Pediatric Emergency Care Applied Research Network

management of low-risk infants with ceftriaxone using Boston and Philadelphia criteria [29]. In their study, sensitivity analyses indicated that treatment of low-risk infants with ceftriaxone would not be the preferred strategy if an alternative diagnostic strategy had $\geq 97 \%$ sensitivity. In our sensitivity analysis, the PECARN strategy would be preferred over the Boston strategy if its sensitivity were $\geq 98 \%$. Our study builds on prior work by considering the impact of modern disease prevalence and epidemiology, as well as prediction rules with improved diagnostic accuracy.

By simulating the experience of large patient populations, Markov analyses identify strategies that benefit the most individuals most often and lend themselves to broader interpretations. Our study findings may also inform shared decision-making discussions. We found that most strategies have similar effectiveness (Table 3). Strategies that require cerebrospinal fluid testing or result in more frequent hospitalization are more costly but may provide benefits not measured in this study, depending on individual risk tolerance or preferences. One of the strengths of this study is the separate consideration of the three most common serious bacterial infections in infants. While their presentations can be similar and prior studies have analyzed them as a group, the prognosis and the consequences of misdiagnosis for each are substantially different, particularly for bacteremia and bacterial meningitis [8, 30-34]. Recent investigators have also attempted to separate UTI from bacteremia and bacterial meningitis, using the term invasive bacterial infection for the latter [4, 11-14]. In this study, developing disease specific models allowed for a better understanding of how each rule performed across the spectrum of disease, from the low risk and low costs of UTI to the high risk and high costs of bacterial meningitis. The cost utilization of a new consensus guideline provided by the American Academy of Pediatrics is an additional consideration; at the time of this publication, no study has validated this decision rule to determine its performance characteristics [43].

Our findings are subject to limitations. Older prediction rules, such as the Philadelphia, Rochester and Boston criteria, were developed and validated during a period when invasive bacterial infection had a higher incidence [44]. In contrast, some recent rules, such as the Modified Philadelphia, Aronson, and Step-by-Step 
criteria, may be more reflective of present-day epidemiology but lack external validation. Local practices often do not strictly adhere to published protocols and our model was limited to strategies with published data [4]. We attempted to account for these factors by examining sensitivity and specificity ranges across their calculated 95\% confidence intervals in sensitivity analyses. We did not consider the impact of increased outpatient visits associated with a larger proportion of infants categorized as low-risk. Given that our sensitivity analysis did not demonstrate that the model was sensitive to either the large costs of hospitalization or the relatively low costs of CSF testing, it is unlikely that the additional cost of an outpatient visit would change model outcomes.

We adapted utility values from the literature. Infant health state utilities are poorly defined and understudied [45]. It is possible that an older individual's experience with bacterial infection, lumbar puncture, or hospitalization is different from that of an infant. For this reason, selected utility values were varied over wide ranges. Varying these values did not change favored strategies.

We used a healthcare perspective and, as such, did not evaluate the perspective of families and caregivers, costs of missed or lost employment, or the disutility of caring for an ill child either in the hospital or at home. This, in turn, could bias results toward or against rules associated with higher hospitalization rates. We did not account for inherent risks of hospitalization and medical interventions, including iatrogenic complications and nosocomial infections, and subsequent associated costs. However, these would only increase the costs associated with strategies that require more frequent hospitalization and thereby not change the ultimate findings in our study. Medical costs and willingness-to-pay were based on the U.S. healthcare system and assumptions about the simulated population were drawn primarily from U.S. based data. As such, it is difficult to apply this model to more resource-limited settings. Despite these limitations, this study demonstrates the value associated with application of clincial prediction rules in the emergency setting, and how we can effectively and efficiently evaluate young febrile infants from the perspective of clinicians and health systems.

\section{Conclusion}

In this cost-effectiveness analysis evaluating strategies for the risk-stratification of young febrile infants, we found that the Boston and PECARN clinical prediction rules are economically reasonable strategies compared to alternative strategies when considering outcomes of UTI, bacteremia, and bacterial meningitis. The Boston strategy was more effective and economically reasonable for bacteremia and bacterial meningitis, whereas the PECARN strategy was preferred in UTI. Our findings highlight the benefits of a risk-stratification strategies that avoid potentially unnecessary hospitalizations, either with empiric antibiotic treatment or by maximizing sensitivity and specificity of the initial evaluation.

\section{Abbreviations \\ SBI: Serious bacterial infection; PECARN: Pediatric Emergency Care Applied Research Network; UTI: Urinary tract infection; ED: Emergency department; QALY: Quality-adjusted life-years; ICER: Incremental cost-effectiveness ratio; UA: Urinalysis; CBC: Complete blood count; CSF: Cerebrospinal fluid; CRP: C-reactive protein; PCT: Procalcitonin; ANC: Absolute neutrophil count.}

\section{Acknowledgements}

The authors thank Dr. Robert Hickey for his valuable insights and suggestions regarding model output interpretation and manuscript revisions.

\section{Authors' contributions}

KAN and KJS conceptualized and designed the study, constructed the model, conducted analyses, and drafted the initial manuscript. SR conceptualized and designed the study, critically appraised model outcomes, and revised the manuscript for intellectually important content. NSR and JD assisted with designing the study, critically appraised model outcomes, and revised the manuscript for intellectually important content. All authors approved the final manuscript as submitted and agree to be accountable for all aspects of the work. This work was presented at the American Academy of Pediatrics National Conference and Exhibition October 4, 2020 and at the October 2020 meeting of the Society for Medical Decision Making.

\section{Funding}

This work was supported by National Institutes of Health [1T32HL134615-01]. The sponsor was not involved in study design; collection, analysis and interpretation of the data; writing the report; or the decision to submit findings for publication

\section{Availability of data and materials}

All data generated or analyzed during this study are included in this published article [and its supplementary information files].

\section{Declarations}

Ethics approval and consent to participate

Institutional review board approval was not required for this study that did not use human participants.

\section{Consent for publication}

Not applicable.

\section{Competing interests}

The authors KAN, SR, NSR, JD, and KJS declare no competing interests.

\section{Author details \\ ${ }^{1}$ Department of Pediatrics, University of Pittsburgh Medical Center, Children's Hospital of Pittsburgh, 4401 Penn Ave, Pittsburgh, PA 15224 60611, USA. ${ }^{2}$ Division of Emergency Medicine, Ann \& Robert H. Lurie Children's Hospital of Chicago, Northwestern University Feinberg School of Medicine, $225 \mathrm{E}$. Chicago Ave, Chicago, IL 606111, USA. ${ }^{3}$ Department of Medicine, University of Pittsburgh Medical Center, 200 Meyran Ave, Pittsburgh, PA 15213, USA.}

Received: 12 April 2021 Accepted: 1 December 2021

Published online: 03 February 2022

\section{References}

1. McCaig L, Nawar E. National hospital ambulatory medical care survey: 2004 emergency department summary. Adv Data. 2006;372:1-29.

2. Niska R, Bhuiya F, Xu J. National Hospital Ambulatory Medical Care Survey: 2007 emergency department summary, vol. 26. Hyattsville: National health statistics reports; 2010. 
3. Nelson D, Walsh K, Fleisher G. Spectrum and frequency of illness presenting to a pediatric emergency department. Pediatrics. 1992;90(1):5-10.

4. Aronson P, Thurm C, Alpern E, Alessandrini E, Williams D, Shah S, et al. Variation in Care of the Febrile Young Infant, 90 days in US pediatric emergency departments (pediatrics (2014) 134:4 (667-677)). Pediatrics. 2015;135(4):775.

5. Biondi E, Evans R, Mischler M, Bendel-Stenzel M, Horstmann S, Lee V, et al. Epidemiology of bacteremia in febrile infants in the United States. Pediatrics. 2013:132(6):990-6.

6. Blaschke AJ, Korgenski EK, Wilkes J, Presson AP, Thorell EA, Pavia AT, et al. Rhinovirus in febrile infants and risk of bacterial infection. Pediatrics. 2018;141(2):1-9.

7. Byington CL, Reynolds CC, Korgenski K, Sheng X, Valentine KJ, Nelson RE, et al. Costs and infant outcomes after implementation of a care process model for febrile infants. Pediatrics. 2012;130(1):e16-24.

8. Klinger G, Chin CN, Beyene J, Perlman M. Predicting the outcome of neonatal bacterial meningitis. Pediatrics. 2000;106(3):477-82.

9. Mintegi S, Gomez B, Carro A, Diaz H, Benito J. Invasive bacterial infections in young afebrile infants with a history of fever. Arch Dis Child. 2018;103(7):665-9.

10. Deangelis C, Joffe A, Wilson M, Willis E. latrogenic risks and financial costs of hospitalizing febrile infants. Am J Dis Child. 1983;137(12):1146-9.

11. Gomez B, Mintegi S, Bressan S, Da Dalt L, Gervaix A, Lacroix L. Validation of the "step-by-step" approach in the management of young febrile infants. Pediatrics. 2016;138(2):e20154381.

12. Kuppermann N, Dayan PS, Levine DA, Vitale M, Tzimenatos L, Tunik $M G$, et al. A clinical prediction rule to identify febrile infants 60 days and younger at low risk for serious bacterial infections. JAMA Pediatr. 2019:173:342-51.

13. Aronson PL, Wang ME, Shapiro ED, Shah SS, DePorre AG, McCulloh RJ, et al. Risk stratification of febrile infants $\leq 60$ days old without routine lumbar puncture. Pediatrics. 2018;142(6):e20181879.

14. Aronson PL, Shabanova V, Shapiro ED, Wang ME, Nigrovic LE, Pruitt CM, et al. A prediction model to identify febrile infants $\leq 60$ days at low risk of invasive bacterial infection. Pediatrics. 2019:144(1):e20183604.

15. Dagan R, Powell KR, Hall CB, Menegus MA. Identification of infants unlikely to have serious bacterial infection although hospitalized for suspected sepsis. J Pediatr. 1985;107(6):855-60.

16. Baskin MN, O'Rourke EJ, Fleisher GR. Outpatient treatment of febrile infants 28 to 89 days of age with intramuscular administration of ceftriaxone. J Pediatr. 1992;120(1):22-7.

17. Baker MD, Bell L, Avner JR. Outpatient mannagmeent without antibiotics of fever in selected infants. N Engl J Med. 1993;329(20):1437-41.

18. Nigrovic LE, Mahajan PV, Blumberg SM, Browne LR, Linakis JG, Ruddy RM, et al. The Yale observation scale score and the risk of serious bacterial infections in febrile infants. Pediatrics. 2017;140(1):e20170695.

19. Garra G, Cunningham SJ, Crain EF. Reappraisal of criteria used to predict serious bacterial illness in febrile infants less than 8 weeks of age. Acad Emerg Med. 2005;12(10):921-5.

20. Jaskiewicz JA, McCarthy CA, Richardson AC, White KC, Fisher DJ, Powell KR, Dagan R, Febrile Infant Collaborative Study Groups. Febrile infants at low risk for serious bacterial infection — an appraisal of the Rochester criteria and implications for management. Pediatrics. 1994;94(3):390-6.

21. Baker MD, Bell LM. Unpredictability of serious bacterial illness in febrile infants from birth to 1 month of age. Arch Pediatr Adolesc Med. 1999;153(5):508-11.

22. Kaplan RL, Harper MB, Baskin MN, Macone AB, Mandl KD. Time to detection of positive cultures in 28-to 90-day-old febrile infants. Pediatrics. 2000;106(6):e74.

23. Velasco R, Gomez B, Benito J, Mintegi S. Accuracy of PECARN rule for predicting serious bacterial infection in infants with fever without a source. Arch Dis Child. 2021;106(2):143-8.

24. Hoshi SL, Kondo M, Okubo I. Economic evaluation of immunisation programme of 23-valent pneumococcal polysaccharide vaccine and the inclusion of 13-valent pneumococcal conjugate vaccine in the list for single-dose subsidy to the elderly in Japan. PLoS One. 2015;10(10):1-16.

25. Handels RLH, Wimo A, Dodel R, Kramberger MG, Visser PJ, Molinuevo JL, et al. Cost-utility of using Alzheimer's disease biomarkers in cerebrospinal fluid to predict progression from mild cognitive impairment to dementia. J Alzheimers Dis. 2017;60(4):1477-87.
26. CPI Inflation Calculator [Internet]. CPI INflation Calculator. 2019. p. 1-2. Available from: https://data.bls.gov/cgi-bin/cpicalc.pl.

27. Clinical Laboratory Fee Schedule 2016 [Internet]. Centers for Medicare \& Medicaid Services. 2019. Available from: https://www.cms.gov/Medicare/ Medicare-Fee-for-Service-Payment/ClinicalLabFeeSched/index.html.

28. Shiell A, Donaldson C, Mitton C, Currie G. Health economic evaluation. J Epidemiol Community Health. 2002;56(2):85-8.

29. Lieu T, Baskin M, Schwartz J, Fleischer G. Clinical and cost-effectiveness of outpatient strategies for managment of febrile infants. Pediatrics. 1992;89(8):1135-44

30. Tsai MH, Hsu JF, Chu SM, Lien R, Huang HR, Chiang MC, et al. Incidence, clinical characteristics and risk factors for adverse outcome in neonates with late-onset sepsis. Pediatr Infect Dis J. 2014;33(1):7-13.

31. Hui C, Neto G, Tsertsvadze A, Yazdi F, Tricco A, Tsouros S, Skidmore B,Daniel R. Diagnosis and Management of Febrile Infants ( $0-3$ months). EvidenceReport/Technology Assessment No. 205 (Prepared by the University of Ottawa Evidence-basedPractice Center under Contract No. HHSA 290-2007-10059-I.) AHRQ Publication No. 12-E004-EF. Rockville, MD: Agency for Healthcare Research and Quality. March 2012.http:// www.ahrq.gov/clinic/epcix.htm.

32. Nadel S, Britto J, Booy R, Maconochie I, Habibi P, Levin M. Avoidable deficiencies in the delivery of health care to children with meningococcal disease. J Accid Emerg Med. 1998;15(5):298-303.

33. Schnadower D, Kuppermann N, Macias CG, Freedman SB, Baskin MN, Ishimine $P$, et al. Febrile infants with urinary tract infections at very low risk for adverse events and bacteremia. Pediatrics. 2010;126(6):1074-83.

34. Nugent J, Childers M, Singh-Miller N, Howard R, Allard R, Eberly M. Risk of meningitis in infants aged 29 to 90 days with urinary tract infection: a systematic review and Meta-analysis. J Pediatr. 2019;212:102-110.e5 Available from: https://doi.org/10.1016/j.jpeds.2019.04.053.

35. Honkinen O, Jahnukainen T, Mertsola J, Eskola J, Ruuskanen O. Bacteremic urinary tract infection in children. Pediatr Infect Dis J. 2000;19(7):630-4.

36. Craig W. Meningitis in the newborn. Arch Dis Child. 1936;64:171-86.

37. Healthcare Cost and Utilization Project [Internet]. U.S. Department of Health and Human Services. 2019. p. https://hcupnet.ahrq.gov/. Available from: https://hcupnet.ahrq.gov/

38. Arias E, Xu JQ. United States life tables, 2017.National Vital Statistics Reports; vol 68 no 7.Hyattsville: National Center for HealthStatistics. 2019.

39. Hart RJ, Stevenson MD, Smith MJ, Scott LJA, Cross K. Cost-effectiveness of strategies for offering influenza vaccine in the pediatric emergency department. JAMA Pediatr. 2018;172(1):e173879.

40. Sanchez-Luna M, Burgos-Pol R, Oyagüez I, Figueras-Aloy J, Sánchez-Solís M, Martinón-Torres F, et al. Cost-utility analysis of Palivizumab for respiratory syncytial virus infection prophylaxis in preterm infants: update based on the clinical evidence in Spain. BMC Infect Dis. 2017;17(1):1-11.

41. Sanders G, Neumann P, Basu A, Brock D, Feeny D, Krahn M, et al. Recommendations for conduct, methodological practices, and reporting of cost-effectiveness analyses: second panel on cost-effectiveness in health and medicine. J Am Med Assoc. 2016;316(10):1093-103.

42. Stinnett AA, Mullahy J. Net health benefits: a new framework for the analysis of uncertainty in cost-effectiveness analysis. Med Decis Mak. 1998;18(2 SUPPL):68-80.

43. Pantell RH, Roberts KB, Adams WG, Dreyer BP, Kuppermann N, O'Leary ST, Okechukwu K, Woods CR Jr; SUBCOMMITTEE ON FEBRILE INFANTS. Evaluation and Management of Well-Appearing Febrile Infants 8 to 60 Days Old. Pediatrics. 2021;148(2):e2021052228.

44. Greenhow TL, Hung Y-Y, Herz A, Losada E, Pantell RH. The changing epidemiology of serious bacterial infections in young infants. Pediatr Infect Dis J. 2014;33(6):595-600.

45. Prosser LA, Corso PS. Measuring health-related quality of life for child maltreatment: a systematic literature review. Health Qual Life Outcomes. 2007:5:1-10

\section{Publisher's Note}

Springer Nature remains neutral with regard to jurisdictional claims in published maps and institutional affiliations. 